\title{
Antenatal contraception counselling and provision of contraception after delivery for first-time young mothers enrolled with a Family Nurse Partnership programme
}

\author{
Bridget Gallagher (D) ,' Sharon T Cameron, ${ }^{2,3}$ Alison Craig, ${ }^{2}$ \\ Annette Gallimore (D) , ${ }^{4}$ Fatim Lakha ${ }^{5}$
}

\begin{abstract}
${ }^{1}$ Family Nurse Partnership, NHS Lothian, Edinburgh, UK

${ }^{2}$ Chalmers Centre, Edinburgh, UK

${ }^{3}$ Obstetrics and Gynaecology, Royal Infirmary of Edinburgh,

Edinburgh, UK

${ }^{4}$ Public Health, NHS Lothian,

Edinburgh, UK

${ }^{5}$ Honorary Consultant in Public Health, NHS Lothian, Edinburgh, UK
\end{abstract}

\section{Correspondence to}

Bridget Gallagher, Family Nurse Partnership, NHS Lothian, Edinburgh EH1 3EG, UK; bridget. gallagher@hotmail.co.uk

Received 24 August 2018 Revised 3 July 2019 Accepted 21 July 2019 Published Online First 7 August 2019

\section{A) Check for updates}

(c) Author(s) (or their employer(s)) 2019. No commercial re-use. See rights and permissions. Published by BMJ.

To cite: Gallagher B, Cameron ST, Craig A, et al. BMJ Sex Reprod Health

2019:45:243-248.

\section{ABSTRACT}

Objective To evaluate antenatal contraceptive counselling and provision of postpartum contraception on contraceptive choices of firsttime teenage mothers enrolled with a Family Nurse Partnership (FNP) programme.

Design Health service research evaluation. Population Cohort of FNP clients $(n=195)$, Scotland, UK.

Methods Antenatal contraceptive counselling and provision of chosen method from the hospital. Evaluation consisted of (i) selfadministered questionnaires of FNP clients and (ii) interviews with FNP clients and FNP nurses.

Main outcome measures FNP client views on intervention. Secondary outcomes included: contraceptive choice antenatally, at day 10 postpartum, 3 and 12 months after delivery, and views of FNP nurses on the intervention.

Results Antenatal questionnaires were completed by 118/195 (61\%) clients. 96/118 $(81 \%)$ agreed that it was very or quite helpful to receive antenatal contraceptive counselling and 80/118 (68\%) were planning to use a long-acting reversible method of contraception (LARC). 97/121 (80\%) wished to receive contraception before leaving the hospital. 104/195 (53\%) completed a questionnaire at day 10 postpartum, of which 33 (32\%) indicated that they had received contraception from the hospital. FNP nurses expressed frustration when contraception was not provided; this was usually attributed to the busy workload of the maternity department.

Conclusions Antenatal contraceptive counselling was appreciated by FNP clients and they expressed a preference for contraception provision following delivery. Over two-thirds planned to use a LARC method but many

\section{Key messages}

- Young women enrolled in a Family Nurse Partnership (FNP) programme appreciate antenatal contraceptive counselling and provision of contraception following delivery.

- Following antenatal contraceptive counselling, more than two-thirds of FNP clients expressed a preference for a long-acting reversible contraception (LARC) method postpartum.

- Increasing the range of contraceptive providers available to FNP clients in the postpartum period may help overcome the gap in access to effective contraception at this time.

did not receive this from the hospital. Further interventions are required to ensure that the provision of postpartum contraception is prioritised for this group of young women.

\section{INTRODUCTION}

The teenage pregnancy rate in Scotland fell 43.8\% between 2007 and 2014 from 57.7 to 32 per 1000 women. ${ }^{1}$ In spite of this, the rate still remains higher than most other Western European countries. There is good evidence that for a teenage mother, a further teenage birth can impact adversely on health, educational attainment and employment opportunities for the mother, resulting in a vicious cycle of poverty for her and her family. ${ }^{2}$ If the subsequent pregnancy occurs after a short interpregnancy interval $(<12$ months between birth and next conception) then that also increases the risk of poor 
obstetric outcomes such as preterm birth, stillbirth and neonatal death. ${ }^{3}$ Reducing unintended teenage pregnancy and repeat teenage pregnancies is a national target for the Scottish Government. ${ }^{4}$ In recognition of the need to prioritise the care of teenage parents and improve their health and well-being, the Scottish Government developed the Pregnancy and Parenthood in Young People Strategy. ${ }^{4}$ Integral to this strategy is the Family Nurse Partnership (FNP) programme, which is an evidence-based, early-intervention, licensed programme involving intensive, structured home visiting whereby a highly-skilled Family Nurse is assigned to a first-time teenage mother from early pregnancy (before 17 weeks' gestation) through to the child's second birthday. ${ }^{5}$ It involves an intensive, structured home visiting programme, aiming to support positive health outcomes for first-time young mothers and their child. In particular; it aims to help young mothers improve their life course by enabling them to identify their own future goals, one of which is planning for future pregnancies.

The FNP programme includes provision of information on contraception during pregnancy visit number 7 which is around 24-26 weeks' gestation. The Family Nurse uses a style of communication underpinned by motivational interview technique and a strengthsbased approach to assess the client's knowledge and past experience with contraception. ${ }^{6}$ Family Nurses guide young mothers to enable them to make a decision about a reliable postpartum contraceptive method after birth. The Family Nurse aims to move young women from intention to accessing services postpartum for their preferred method of contraception. However, during the postpartum period the requirements of caring for a young baby and recovering from childbirth can take precedence over accessing contraception for many mothers, exposing women to the risk of an unintended pregnancy. ${ }^{7}$

In 2014, a pilot project named APPLES (Access to Post Partum LARC in Edinburgh South) was conducted in the Edinburgh region. ${ }^{8}$ This project piloted routine antenatal contraceptive counselling by community midwives and provision of contraception postnatally from the maternity hospital, with facilitated access to long-acting reversible contraception (LARC).

It was decided to extend the pilot to include a cohort of clients from the local FNP programme as a distinct arm of the project. Thus, in addition to the existing contraceptive discussion included in the FNP programme delivered by Family Nurses, clients also received counselling on contraception (at 22 weeks) from a community midwife. In addition, FNP nurses received contraception updates from a nurse consultant (AC) in sexual and reproductive healthcare (SRH). For the project, there was a steering group that included obstetricians, midwives, SRH specialists, public health and FNP to guide overall project direction and facilitate communication between key stakeholders.
FNP clients' preferred method of contraception was documented in maternity notes at approximately 32 weeks. The chosen contraceptive method was provided where possible (and if medically eligible) following delivery and before discharge from the maternity service. The methods of contraception that could be provided were the progestogen-only pill, implant, injectable and condoms. ${ }^{8}$ For FNP clients who wanted intrauterine contraception (IUC), this was either provided at a planned caesarean section or clients were given an appointment for insertion of the IUC 1 month later at a local SRH clinic. ${ }^{8}$ All contraception and contraceptive services were provided at no cost, as is the norm within the National Health Service (NHS).

We wished to determine (i) views of FNP clients on the intervention (contraceptive counselling and provision of method from maternity hospital), (ii) resultant uptake of effective contraception postpartum and (iii) the views of FNP nurses on the intervention.

\section{METHODS}

Evaluation consisted of self-administered questionnaires of client's views of (i) antenatal contraceptive counselling and planned contraception (at 32 weeks) and (ii) contraceptive choice at day 10 postpartum, 3 and 12 months after delivery and (iii) in-depth interviews with FNP clients and a focus group discussion with Family Nurses about the intervention.

The antenatal questionnaire was the same as that used in the APPLES project and consisted of tick box answers to questions on contraception, basic demographics and reproductive history. ${ }^{8}$ Questionnaires used postpartum and at 3 at 12 months were similar but also included a question on ethnicity. In addition, the 3 and 12 months questionnaire also requested the respondent's postal area of residence to generate the Scottish Index of Multiple Deprivation (SIMD). ${ }^{9}$ FNP nurses distributed questionnaires to clients for self-completion. Completed questionnaires were placed in a sealed, opaque envelope and collected by FNP nurses. Analysts entered the data from the questionnaires into a secure database conforming to NHS confidentiality standards. Descriptive statistics were produced.

Face-to-face interviews were conducted with five purposively selected FNP clients identified by the FNP service manager as a representative sample from the local FNP cohort. Included in this sample was a client from the lower age bracket (age 16 years and under), a minority ethnic client, and mothers from different socioeconomic backgrounds. A focus group was held with the Family Nurses $(n=8)$ about their views on intervention. The qualitative researcher (AG) conducted interviews and the focus group discussion at health centre sites. These lasted approximately 40 mins and were audio-recorded. A topic guide, based on the research questions, outlined the key areas to be covered. These were transcribed verbatim and inductive thematic analysis was undertaken. AG generated 
Table 1 Demographics of respondents

\begin{tabular}{|c|c|c|c|c|}
\hline \multicolumn{5}{|l|}{ Age group at EDD (years) } \\
\hline Unknown* & \multicolumn{4}{|l|}{$29(15 \%)$} \\
\hline $19-20$ & \multicolumn{4}{|l|}{$108(56 \%)$} \\
\hline $16-18$ & \multicolumn{4}{|l|}{$50(27 \%)$} \\
\hline $13-15$ & \multicolumn{4}{|l|}{$5(2 \%)$} \\
\hline Ethnicity & Antenatal & Day 10 & 3 months & 1 year \\
\hline British & $t$ & $89(85 \%)$ & $81(83 \%)$ & $56(82 \%)$ \\
\hline Eastern/Central Europe & $\dagger$ & $4(4 \%)$ & $2(2 \%)$ & $2(3 \%)$ \\
\hline South and East Asian & $t$ & $0(0 \%)$ & $2(2 \%)$ & $1(1 \%)$ \\
\hline American & $\dagger$ & $0(0 \%)$ & $1(1 \%)$ & $1(1 \%)$ \\
\hline African & $\dagger$ & $1(1 \%)$ & $0(0 \%)$ & $2(3 \%)$ \\
\hline Caribbean & $\dagger$ & $1(1 \%)$ & $0(0 \%)$ & $0(0 \%)$ \\
\hline Not stated/other & $\dagger$ & $9(9 \%)$ & $12(12 \%)$ & $7(10 \%)$ \\
\hline \multicolumn{5}{|l|}{ SIMD deprivation quintile } \\
\hline 1 - Most deprived & $\dagger$ & $\dagger$ & $50(51 \%)$ & $40(57 \%)$ \\
\hline 2 & $\dagger$ & $\dagger$ & $20(21 \%)$ & $8(13 \%)$ \\
\hline 3 & $\dagger$ & $\dagger$ & $10(10 \%)$ & $5(7 \%)$ \\
\hline 4 & $\dagger$ & $\dagger$ & $10(10 \%)$ & $7(10 \%)$ \\
\hline 5 - Least deprived & $\dagger$ & $\dagger$ & $3(3 \%)$ & $2(3 \%)$ \\
\hline Unknown & $\dagger$ & $\dagger$ & $5(5 \%)$ & $7(10 \%)$ \\
\hline
\end{tabular}

*Data not completed.

tData not collected.

EDD, estimated delivery date; SIMD, Scottish Index of Multiple Deprivation.

codes on the basis of repeated reading of the transcripts for significant themes. ${ }^{10}$ Data were organised by cross-sectional indexing using QSR NVivo 10 and an inductive approach was adopted.

The local ethics committee officer reviewed the project protocol, surveys and topic guides and confirmed that ethical committee approval was not required for this health services research.

\section{Patient involvement}

Patients were not directly involved in the design or conduct of this study.

\section{RESULTS}

Questionnaires were distributed to all 195 FNP women. The response rate to the questionnaires was $61 \%(118 / 195)$ at 32 weeks gestation, 53\% (104/195) at day 10 postpartum, $50 \%(98 / 195)$ at 3 months and $35 \%(69 / 195)$ at 12 months following delivery.

Table 1 shows the demographics of respondents.

\section{Antenatal questionnaire}

Of the 118 respondents, 94\% (111/118) indicated they had discussed contraception antenatally and $81 \%$ (96/118) reported they found the discussion either 'very' or 'quite helpful'. Some 81\% (95/118) of respondents indicated that they found the timing of the contraceptive discussion with the midwife (at 22 weeks) 'about right', with 12\% (14/118) 'unsure', three $(3 \%)$ thinking it was too early, one $(1 \%)$ too late, and five (4\%) left this question unanswered. The contraceptive choices made antenatally are shown in table 2. Very few women $(n=2)$ were unsure about what method they planned to use and $68 \%$ of respondents (80/118) intended to use a LARC method postpartum (intrauterine contraception, implant or injectable). The most popular choice was the implant. The majority of the women $(80 \%, n=97)$ said they would want their preferred method of contraception given prior to leaving the hospital.

In response to a question on future pregnancy intentions within 1 year after delivery, no FNP clients indicated that they intended to try for a pregnancy, $17 \%(20 / 118)$ indicated that they felt their family was complete and $51 \%(60 / 118)$ were undecided as to their intention. The remaining 30\% (36/118) intended to try for another baby but not within 1 year. Two women did not answer this question.

\section{Questionnaire - day 10 postpartum}

The planned method of contraception chosen by women at day 10 postpartum is shown in table 2 .

Some 67/104 (64\%) respondents did not receive any contraception before discharge from hospital. Of the women who wanted an intrauterine method $(n=9)$, only one had a future appointment at a community sexual health service for fitting a device. 
Table 2 Contraceptive choice antenatally, at day 10, 3 months and 1 year after delivery.

\begin{tabular}{|c|c|c|c|c|}
\hline Contraceptive method & $\begin{array}{l}\text { Antenatal }(n) \\
(n=118 / 195,60 \%)\end{array}$ & $\begin{array}{l}\text { Day } 10(n) \\
(n=104 / 195,53 \%)\end{array}$ & $\begin{array}{l}3 \text { months }(n) \\
(n=98 / 195,50 \%)\end{array}$ & $\begin{array}{l}1 \text { year }(n) \\
(n=69 / 195,35 \%)\end{array}$ \\
\hline CU-IUD & 7 & 3 & 1 & 0 \\
\hline LNG-IUS & 12 & 6 & 0 & 2 \\
\hline Implant & 57 & 51 & 35 & 36 \\
\hline Injectable & 14 & 13 & 10 & 4 \\
\hline Combined pill & 16 & 17 & 16 & 9 \\
\hline Progestogen-only pill & 12 & 9 & 7 & 3 \\
\hline Combined patch & 1 & 1 & 1 & 5 \\
\hline Condoms & 11 & 7 & 15 & 10 \\
\hline Other & 1 & 0 & 3 & 0 \\
\hline Not sure & 9 & 6 & 0 & 0 \\
\hline None & 1 & 2 & 16 & 9 \\
\hline
\end{tabular}

Women could choose more than one option for these questions therefore the numbers will not add up to the total.

Cu-IUD, copper-bearing intrauterine device; LNG-IUS, levonorgestrel-releasing intrauterine system.

\section{Questionnaire - 3 months and 12 months}

Table 2 shows the method of contraception that women were using at 3 and 12 months postpartum. The implant remained the most commonly used method and most respondents at both time points were using an effective method (other than condoms). Reported use of LARC by respondents was 46/98 (47\%) and 42/69 (61\%) at 3 and 12 months, respectively (table 2 ).

\section{Views of FNP clients and FNP nurses}

Qualitative data showed that FNP clients found antenatal contraceptive counselling helpful, and appreciated it when contraception was provided at the hospital rather than them having to wait several weeks to receive it.

Obviously, like, we had, we had to speak about these kind of things. Because, obviously, I dinnae want to be falling pregnant, straight after, do you know what I mean. And, yeah, it kind of helped me a lot. Like, I didn't know that, like, I could get it [the implant] put in straightaway after I had, I had...I thought I had to wait, like, a couple of weeks.

\section{FNP Client 5 talking about antenatal contraceptive discussion}

So if it's already in [implant] and booked, you don't need to go anywhere. You're already there for the reason... [Yeah] so I think it makes it quite well... and it's there, you...it's all done. You don't need to worry about it any more and when you've just had a baby, I think the last thing on your mind is going to get something like that, so yeah, that...I think that's the best idea.

\section{FNP Client 2 talking about provision of implant after delivery}

Family nurses reported that clients who did not receive their chosen method of contraception were disappointed and the Family Nurses expressed frustration about this given the effort that had been put into counselling women antenatally, although they recognised that the duration of the hospital stay after delivery and maternity staffing levels impacted on a woman's likelihood of receiving contraception.

I think it's time. I think the clients that I've had successfully you know, counselled and they've had, well, it's generally an implant they have done. It really has correlated with their hospital stay. So my clients have stayed more than one night, they have had their contraception as an inpatient before discharge.

\section{FNP Nurse 1 feedback}

It wasn't a priority. I mean the thing is, it's never gonna be a priority in that environment unless they have the staffing levels. Because if you've got a member of staff who's faced with being struck off for not doing postoperative observations or going and having a half hour chat with somebody about contraception that's traditionally been sorted out 6 weeks later, there's no way they're gonna prioritise the second thing.

\section{FNP Nurse 2 feedback}

Family Nurses reported that they were pleased that as a result of participation in the project they were able to expand their knowledge of contraceptive methods as a consequence of receiving additional educational session from the Nurse Consultant.

\section{DISCUSSION}

\section{Main findings}

This study demonstrated that teenage first-time mothers appreciated antenatal contraceptive counselling delivered by community midwives (in addition to that delivered by FNP nurses). Most had decided by the time of the questionnaire at 32 weeks' gestation on a method of contraception that they wished to use postpartum and just over two-thirds planned to use one of the most effective LARC methods of contraception, most commonly 
the implant. It was also clear that within this cohort, most of the women were not planning to become pregnant within the next year. Rather, the preference for LARC methods indicates that they were actively seeking to avoid an unintended pregnancy in the coming year. There is a lack of data on the contraceptive preferences of young mothers after delivery. In larger studies of women in this same geographical region, $43 \%$ of pregnant women expressed a desire for LARC postpartum. ${ }^{811}$ In studies from the USA it has been reported that 23\% of young mothers plan to use an implant or intrauterine method. ${ }^{12}$ A figure of $68 \%$ choosing LARC does therefore seem high. Given that this was not a comparative study, it is impossible to determine how much of these contraceptive preferences were due to antenatal contraceptive counselling from the midwife and FNP nurse, or the relative effect of counselling by each of these groups. FNP clients expressed a clear preference to receive their chosen contraceptive method from the hospital before being discharged home. This is important as it provides some evidence of the acceptability of this contraceptive approach among young mothers. All mothers struggle to make clinic visits in the postpartum period, and there is some evidence that young mothers may face particular difficulties. $^{7}$ Although just one-third of clients received contraception immediately after delivery, this still represents a significant proportion of women benefitting from facilitated access to effective contraception at this time. This should be viewed in the context that before this study, no women were provided with contraception in the maternity setting.

LARC methods require availability of a trained provider. During this study the implant was provided by obstetric staff only, which together with short hospital stays were reported in the APPLES study and this study to be major barriers to provision in a busy maternity setting. ${ }^{8}$ Both FNP clients and nurses were frustrated when contraception was not provided from the hospital. Expanding the number and range of trained implant fitters to include hospital midwives could be a strategy to help meet the demand for this popular choice of method in the maternity setting. In addition, initiatives such as community midwives being trained to provide implants to women at home in the postpartum period is another possible option for improving access to this method. ${ }^{13}$

\section{Strengths and weaknesses}

The study's strengths are that it was conducted in a well-defined cohort of young, first-time, teenage mothers enrolled with FNP and that it reports on contraceptive choice and method use longitudinally over 1 year following delivery in this group. There is a paucity of information on the views of teenage mothers on postpartum contraceptive choices and preferences for provision and so this article adds to the evidence on which future service development could be based. The mixed methods nature of the study is another strength as the qualitative research adds context to the choices women made and received. The study was a small one and set in a single geographical area of the UK, so the findings may not be representative of other FNP client populations, nor of the views of FNP nurses elsewhere. In addition, while completion of questionnaires antenatally was high, rates declined over the course of the 12 months post-delivery to a response rate of just over one third, which reduced the robustness of the data. Although the study reports on contraceptive preference and uptake, it was not a randomised controlled trial and so we cannot determine whether or the degree to which the reported use of methods was influenced by the antenatal counselling. In addition, the study is not able to link data on contraceptive use with subsequent pregnancies.

\section{What this study adds to the literature}

Previous studies have shown that delaying contraception until a 6-week postnatal visit to a general practitioner may be too late, as fertility and sexual activity can resume before this time. Also, there is some evidence to suggest that teenage mothers may be a group who are less likely to attend this postnatal visit and so are less likely to access effective contraception. ${ }^{7}$ Access to contraception for young mothers is highly dependent on health services being responsive to their needs. Evidence from the USA and Australia indicates that teenage mothers who choose to receive a LARC method after delivery have a significant reduction in the risk of another pregnancy in the next year compared with counterparts choosing pills, condoms and other methods. ${ }^{14}{ }^{15}$ Guidelines from the Faculty of Sexual \& Reproductive Healthcare (FSRH) in the UK (endorsed by the Royal Colleges of Midwives, General Practitioners and Obstetricians and Gynaecologists) recommend that all women (including teenagers) should have routine antenatal counselling. ${ }^{16}$ The guidelines also recommend that women should be advised that LARC are the most effective methods and that immediate provision postpartum should be available if women choose this and are medically eligible for these methods. ${ }^{15}$ This project involved close collaboration between key stakeholder groups and support from senior hospital management throughout, and this is essential for the successful implementation of such postpartum contraception services.

\section{Future research}

Involvement in this study provided FNP nurses with the opportunity to work more closely with SRH and maternity services as a result of participating in this project. The data generated provided insight into subsequent pregnancy intention and contraceptive choices in this cohort of clients. In addition, FNP nurses were also able to expand their knowledge of contraception as a result of the update they received from the Nurse Consultant. Future areas for FNP programme development could include developing further the contraceptive role of the FNP nurse to include provision of contraception. This could build on the trusting relationship between 
the FNP clients and their nurses and possibly facilitate uptake of effective methods since the frequent interactions between the clients and nurses offers more opportunities for the provision of a contraceptive method. The FSRH recommends that the range of healthcare providers involved in caring for women during pregnancy provide opportunities to discuss contraception and facilitate a woman's access to ongoing contraception. ${ }^{16}$ Given that a change in the role of the FNP nurse from providing support around contraception to being a provider may also potentially harm individual relationships, a potential model could involve a discrete number of FNP nurses who would be the contraceptive providers for the entire team. We plan to evaluate this approach in a future study.

\section{CONCLUSIONS}

Young women enrolled in this FNP appreciated antenatal contraceptive counselling and expressed a clear preference for receiving a contraceptive method after delivery. More than two-thirds expressed a preference for a LARC method. Just one-third of all clients received a contraceptive method, and this was attributed to the hospital staff's busy workloads and short stays within the maternity department. Increasing the range of contraceptive providers available to FNP clients in the immediate postpartum period could help address the gap in access to effective contraception for these women at this time. This may be an effective strategy to prevent unintended pregnancies and optimise birth spacing, thus improving the health and life opportunities for young mothers and their babies.

Acknowledgements The support of Danielle Ene (Senior IT Analyst, NHS Lothian) with database management and analysis is gratefully acknowledged, as is the support of the APPLES steering committee and community and hospital maternity staff and the Family Nurse Partnership (FNP) NHS Lothian.

Contributors BG conducted the study and was responsible for study conduct, analysing and writing drafts. AG was responsible for qualitative components. STC, AC and FL contributed to study design, analysis and drafts. All authors approved the final draft. Details of ethical approval: none required. The NHS Lothian ethics committee officer reviewed the project protocol, surveys and topic guides and confirmed that ethical committee approval was not required for this health services research.

Funding This study received a grant from Edinburgh and Lothians Health Foundation.

Competing interests None declared.

Patient consent for publication Not required.

Provenance and peer review Not commissioned; externally peer reviewed.

Data availability statement Data are available upon reasonable request. All data relevant to the study are included in the article or uploaded as supplementary information.

ORCID iDs

Bridget Gallagher http://orcid.org/0000-0002-7160-718X
Annette Gallimore http://orcid.org/0000-0002-0715-8594

\section{REFERENCES}

1 National Services Scotland Information Services Division. Year of conception ending 31 December 2014, 2016. Available: https://www.isdscotland.org/Health-Topics/Sexual-Health/ Publications/2016-07-05/2016-07-05-TeenPreg-Report.pdf [Accessed 30 June 2018].

2 Baldwin MK, Edelman AB. The effect of long-acting reversible contraception on rapid repeat pregnancy in adolescents: a review. J Adolesc Health 2013;52:S47-S53.

3 Smith GCS, Pell J, Dobbie R. Interpregnancy interval and risk of preterm birth and neonatal death: retrospective cohort study. BMJ 2003;327.

4 Scottish Government. Consultation on the pregnancy and parenthood in young people strategy. Available: http://www.gov. scot/Publications/2015/07/3144 [Accessed 30 June 2018].

5 Olds DL. Prenatal and infancy home visiting by nurses: from randomized trials to community replication. Prev Sci 2002;3:153-72.

6 Channon S, Bekkers M-J, Sanders J, et al. Motivational interviewing competencies among UK family nurse partnership nurses: a process evaluation component of the building blocks trial. BMC Nurs 2016;15:55.

7 Lunniss H, Chen E, Cameron ST. Views of general practitioners towards postpartum contraception; a qualitative study. J Fam Plann Reprod Health Care 2016;42:99-106.

8 Cameron ST, Craig A, Sim J, et al. Feasibility and acceptability of introducing routine antenatal contraceptive counselling and provision of contraception after delivery: the APPLES pilot evaluation. BJOG 2017;124:2009-15.

9 Scottish Government. Scottish Index of Multiple Deprivation, 2006. Available: http://www.gov.scot/Topics/Statistics/SIMD [Accessed 30 June 2018].

10 Braun V, Clarke V. Using thematic analysis in psychology. Qual Res Psychol 2006;3:77-101.

11 Heller R, Cameron S, Briggs R, et al. Postpartum contraception: a missed opportunity to prevent unintended pregnancy and short inter-pregnancy intervals. J Fam Plann Reprod Health Care 2016;42:93-8.

12 Chacko MR, Wiemann CM, Buzi RS, et al. Choice of postpartum contraception: factors predisposing pregnant adolescents to choose less effective methods over long-acting reversible contraception. J Adolesc Health 2016;58:628-35.

13 Croan L, Craig A, Scott L, et al. Increasing access to contraceptive implants in the postnatal period via a home insertion service by community midwives [J Fam Plann Reprod Health Care. 2017 Nov 8. pii: jfprhc-2017-101749]. BMJ Sex Reprod Health 2018;44:61-4.

14 Damle LF, Gohari AC, McEvoy AK, et al. Early initiation of postpartum contraception: does it decrease rapid repeat pregnancy in adolescents? J Pediatr Adolesc Gynecol 2015;28:57-62.

15 Lewis LN, Doherty DA, Hickey M, et al. Predictors of sexual intercourse and rapid-repeat pregnancy among teenage mothers: an Australian prospective longitudinal study. Med J Aust 2010;193:338-42.

16 Faculty of Sexual \& Reproductive Healthcare (FSRH). Contraception after pregnancy, 2017. Available: https://www. fsrh.org/standards-and-guidance/documents/contraception-afterpregnancy-guideline-january-2017/ [Accessed 30 June 2018]. 